\title{
Tubal pregnancy with molar degeneration in concurrent eutopic pregnancy: a case report
}

\author{
Jesus Joaquin Hijona Elosegui* , Antonio Carballo Garcia, Francisco Javier Frutos Arenas, \\ Juan Manuel Torres Marti
}

Department of Obstetrics and Gynecology, Hospital Materno Infantil, Complejo Hospitalario de Jaen, Jaen, Spain. Email: ${ }^{*}$ jesushijona@gmail.com

Received 8 March 2011; revised 11 March 2011; accepted 15 April 2011.

\begin{abstract}
This research paper presents the infrequent case of a heterotopic pregnancy based on a tubal ectopic pregnancy with molar degeneration in concurrent eutopic pregnancy. Treatment with evacuation/suction curettage and perlaparoscopic salpingectomy was required. This case report confirms what is biologically valid in the statistically unlikely.
\end{abstract}

Keywords: Heterotopic Pregnancy; Hydatiform Mole; Molar Pregnancy; Pregnancy Complications; Tubal Pregnancy

\section{INTRODUCTION}

Vaginal bleeding in early pregnancy is the most frequent reason for consultation by pregnant women. Differential diagnosis in these cases is not always easy and ultrasound scan and determination of $\beta$ hCG are frequently necessary to determine the pregnancy feasibility and to discard other bleeding reasons.

The main reasons for metrorrhagia in the first quarter of pregnancy are miscarriage, ectopic pregnancy and gestational trophoblastic disease [1]. Coexistence of these pathologies in the same patient, although infrequent, is possible; thus, it is always convenient to carry out a thorough examination, including detailed case history, full physical examination and a thorough analytic and ultrasound evaluation.

\section{CASE REPORT}

A 32 years old primiparous woman without a significant gynecological case history came to the emergency service in her sixth week of amenorrhea because of a painless, dark and mild metrorrhagia, without any other related symptoms. When palpating, the abdomen was soft and non-tender without masses, megalies, painful areas or peritoneal irritation. The gynecological examination did not reveal any significant findings and transvaginal ultrasound showed a regular uterus with a $25 \mathrm{~mm}$ thick endometrium. It was, hyperechogenic and showed small anechoic images which did not confirm an image of gestational sac. In the right adnexus, an anechoic formation with a diameter of $34 \mathrm{~mm}$ (compatible with a corpus luteum) was observed. In contralateral adnexal area, a similar image with smaller diameter (23 mm), compatible with another follicular formation, was also found. The Douglas cul-de-sac was taken up by a small amount of free liquid. Determination of $\beta$ hCG in serum was $7192 \mathrm{mUI} / \mathrm{ml}$.

Due to these results and in view of the clinical suspicion of a potential hydatiform mole or ectopic pregnancy, it was decided to keep an expectant management with new clinic, analytical and ultrasound control after 48 hours.

24 hours after the first examination, the patient returned to the emergency service with abdominal pain. The abdomen exploration did not show any changes, except for the pain appearance after a deep palpation on the left iliac fossa. The gynecological examination did not reveal any significant modifications (except for the painful mobilization of the cervix) and the transvaginal ultrasound did not show any changes regarding the previous one. The analytical study resulted in a determination of beta-HCG of $8038 \mathrm{mUI} / \mathrm{ml}$. In view of the clinical and analytical progress and taking into consideration the possibility of ectopic pregnancy with a potential concurrent molar degeneration, we decided that the patient should be admitted, but she refused.

72 hours after the second examination, the patient returned to the emergency services because she had experienced a pain increase. The abdominal examination suggested a potential acute abdomen. The determination of $\beta \mathrm{hCG}$ at that moment was $19262 \mathrm{mUI} / \mathrm{ml}$. The ultrasound revealed an endometrium with increased vascular supply and signs suggesting molar degeneration (Figures 1-2), as well as the presence in both ovaries of corpus 


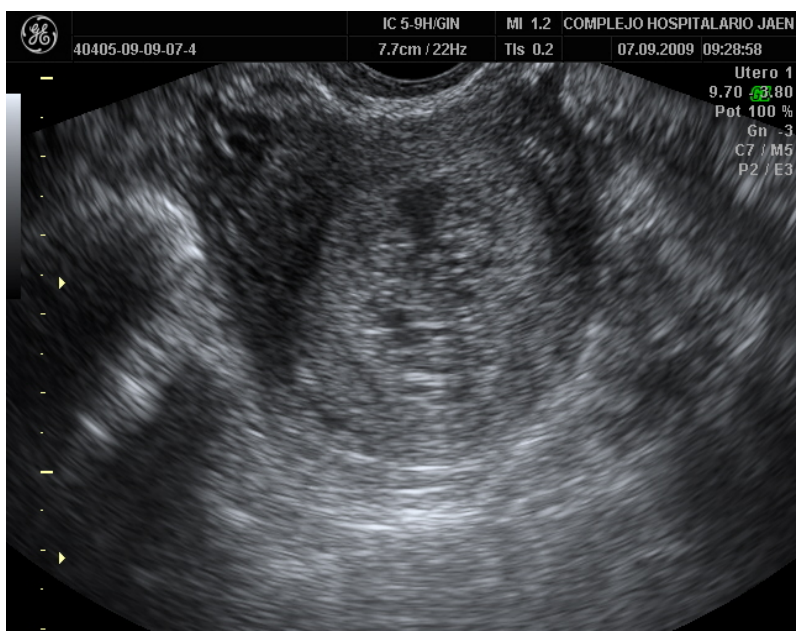

Figure 1. Unstructured uterine cavity suggesting molar degeneration.

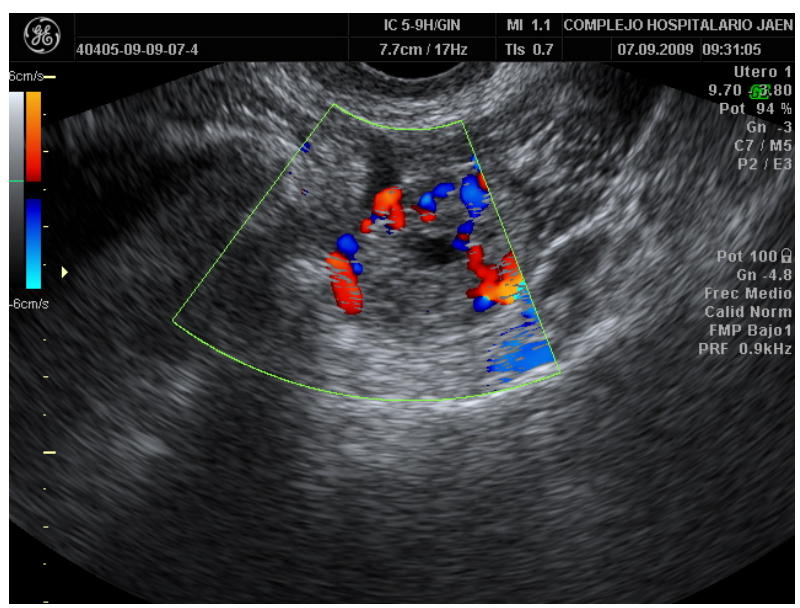

Figrue 3. Corpus luteum and an ypsilateral right adnexal image compatible with a tubal pregnancy.

luteum and a right adnexal image compatible with a tubal pregnancy. These findings (Figures 3-4) strengthened the diagnostic suspicion previously set so we decided to admit the patient for treatment (diagnostic/therapeutic laparoscopy and uterine evacuation). The patient underwent a perlaparoscopic right salpingectomy and evacuation/suction curettage, procedures which were carried out without any incidences. Post-operative course was successfull and determinations of $\beta$ hCG decreased progressively until the date of the current communication (3 months after surgery). The path examination carried out after surgery confirmed both diagnosis.

\section{DISCUSSION}

The term of "heterotopic pregnancy" refers here to the coexistence of an intrauterine pregnancy with another

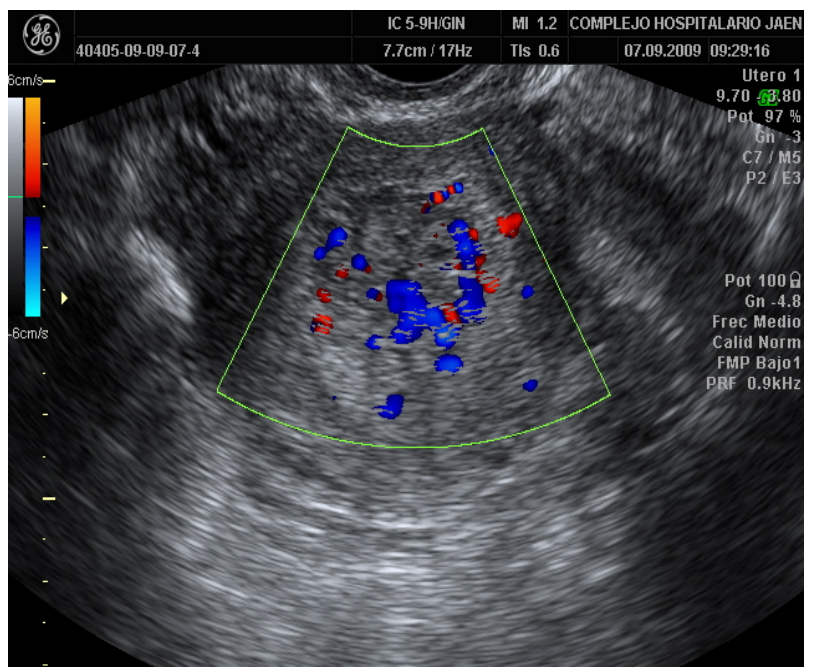

Figure 2. Endometrium with an increased vascular supply.

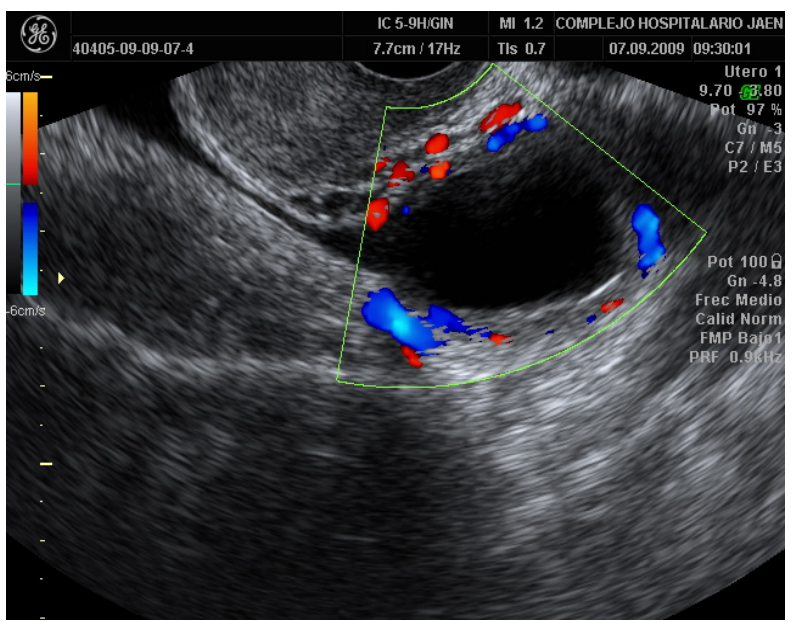

Figure 4. Corpus luteum in left ovarian.

pregnancy located outside the endometrial cavity. The case report we present corresponds to an extremely infrequent variation of this kind of pregnancy, of an unknown prevalence because of the short amount of cases communicated (as far as we know, there is only one case) [1].The therapeutic behavior was simplified due of the unfeasibility of both pregnancies, but in another kind of heterotopic pregnancy it should be individualized and always oriented by the process seriousness and the potential feasibility of the eutopic pregnancy.

\section{REFERENCES}

[1] Nicks, B.A., Fitch, M.T. and Manthey, D.E. (2009) A case of intrauterine molar pregnancy with coexistent ectopic pregnancy. The Journal of Emergency Medicine, 36, 246-249. 